ANNALES

POLONICI MATHEMATICI

$80(2003)$

\title{
Multidimensional analogue of the van der Corput-Visser inequality and its application to the estimation of the Bohr radius
}

\author{
by L. Aizenberg (Ramat-Gan), E. Liflyand (Ramat-Gan) \\ and A. VIDRAS (Nicosia)
}

\begin{abstract}
We present a multidimensional analogue of an inequality by van der Corput-Visser concerning the coefficients of a real trigonometric polynomial. As an application, we obtain an improved estimate from below of the Bohr radius for the hypercone $\mathcal{D}_{1}^{n}=\left\{z \in \mathbb{C}^{n}:\left|z_{1}\right|+\ldots+\left|z_{n}\right|<1\right\}$ when $3 \leq n \leq 10$.
\end{abstract}

1. Bohr radius. The following classical result can be found in [9]: If the power series

$$
\sum_{k=0}^{\infty} c_{k} z_{1}^{k}
$$

converges in the unit disk $\left\{z_{1} \in \mathbb{C}:\left|z_{1}\right|<1\right\}$ and the modulus of its sum is smaller than 1 , then

$$
\sum_{k=0}^{\infty}\left|c_{k} z_{1}^{k}\right|<1
$$

in the disk $\left\{z_{1} \in \mathbb{C}:\left|z_{1}\right|<1 / 3\right\}$ and the constant $1 / 3$ cannot be improved. We now turn to the formulation of multidimensional analogues of this result. Let $\mathcal{D}$ be a complete Reinhardt domain. We denote by $R(\mathcal{D})$ the largest nonnegative number $r$ with the following property: if the power series

$$
\sum_{|\alpha| \geq 0} c_{\alpha} z^{\alpha}
$$

where $\alpha=\left(\alpha_{1}, \ldots, \alpha_{n}\right)$, all $\alpha_{j}$ are nonnegative integers, $z^{\alpha}=z_{1}^{\alpha_{1}} \ldots z_{n}^{\alpha_{n}}$, $|\alpha|=\left|\alpha_{1}\right|+\ldots+\left|\alpha_{n}\right|$, converges in $\mathcal{D}$ and the modulus of its sum is smaller

2000 Mathematics Subject Classification: Primary 42A05, 32A05; Secondary 32A07. Key words and phrases: van der Corput-Visser inequality, Bohr radius, hypercone. 
than 1 , then

$$
\sum_{|\alpha| \geq 0}\left|c_{\alpha} z^{\alpha}\right|<1
$$

in the homothety $r \mathcal{D}$. The number $R(\mathcal{D})$ is called the Bohr radius. We do not know of any bounded domain $\mathcal{D} \subset \mathbb{C}^{n}$ for which the precise value of the Bohr radius is known. However, there are a number of cases where estimates for the Bohr radius from above and from below have been obtained. Such is the case of the estimates in [8]. There, for the unit polydisk

$$
U^{n}=\left\{z \in \mathbb{C}^{n}:\left|z_{j}\right|<1, j=1, \ldots, n\right\},
$$

the following inequalities are proved for $n>1$ :

$$
\frac{1}{3 \sqrt{n}}<R\left(U^{n}\right)<\frac{2 \sqrt{\log n}}{\sqrt{n}} .
$$

This means that $R\left(U^{n}\right)$ tends to 0 as $n \rightarrow \infty$.

In [2] the following estimates for the Bohr radius of the hypercone

$$
\mathcal{D}_{1}^{n}=\left\{z \in \mathbb{C}^{n}:\left|z_{1}\right|+\ldots+\left|z_{n}\right|<1\right\}
$$

are obtained:

$$
\frac{1}{3 \sqrt[3]{e}}<R\left(\mathcal{D}_{1}^{n}\right) \leq \frac{1}{3} .
$$

We point out that unlike the case of the polydisk, this Bohr radius does not tend to 0 as $n \rightarrow \infty$.

Further estimates of the Bohr radius for the domain

$$
\mathcal{D}_{p}^{n}=\left\{z \in \mathbb{C}^{n}:\left|z_{1}\right|^{p}+\ldots+\left|z_{n}\right|^{p}<1\right\}
$$

where $1 \leq p<\infty$, can be found in [7]. Other multidimensional analogues of the Bohr theorem can be found in [3]-[6], [11].

The estimate (1.1) was significantly improved in [1] for the case $n=2$. The main ingredient there was the following result due to J. G. van der Corput and C. Visser ([10]): Let

$$
F(t)=\sum_{j=-N}^{N} a_{j} e^{i j t}
$$

be a real trigonometric polynomial. Then

$$
\left|a_{0}\right|+\left|a_{k}\right|+\left|a_{-k}\right| \leq \max _{0 \leq t \leq 2 \pi}|F(t)|
$$

whenever $k>N / 2$.

We remark here that the function $e^{i N t} F(t)$ is a holomorphic polynomial in $e^{i t}$. This implies that the inequality (1.2) is significantly sharper than the Cauchy estimates. 
In the present article we obtain a multidimensional analogue for the inequality (1.2), which, in turn, improves the estimate (1.1) of the Bohr radius from below for small dimensions $n$.

2. Multivariate van der Corput-Visser inequality. Consider the trigonometric polynomial

$$
F(t)=F\left(t_{1}, \ldots, t_{n}\right)=\sum_{|\alpha| \leq N} a_{\alpha} e^{i\left(\alpha_{1} t_{1}+\ldots+\alpha_{n} t_{n}\right)} .
$$

Denote by $e_{j}=(0, \ldots, 0,1,0, \ldots, 0)$ the unit vector in $\mathbb{R}^{n}$ with all entries zero except the $j$ th one which is 1 . Now, we state the following

THEOREM 2.1. If the trigonometric polynomial (2.1) is real, then

$$
\left|a_{0}\right|+\sum_{j=1}^{n}\left(\left|a_{N e_{j}}\right|+\left|a_{-N e_{j}}\right|\right) \leq \max _{\substack{0 \leq t_{j} \leq 2 \pi \\ j=1, \ldots, n}}|F(t)| .
$$

Proof. The proof is by induction. For $n=1$ this is the inequality (1.2). Assume that (2.2) holds for $n-1$. Then we write the polynomial (2.1) as

$$
F(t)=\sum_{\alpha_{n}=-N}^{N} e^{i \alpha_{n} t} \sum_{\left|\alpha_{1}\right|+\ldots+\left|\alpha_{n-1}\right| \leq N-\left|\alpha_{n}\right|} a_{\alpha} e^{i\left(\alpha_{1} t_{1}+\ldots+\alpha_{n-1} t_{n-1}\right)} .
$$

Applying to $F(t)$ the one-dimensional result, we obtain

$$
\begin{array}{r}
\left|a_{N e_{n}}\right|+\left|a_{-N e_{n}}\right|+\left|\sum_{\left|\alpha_{1}\right|+\ldots+\left|\alpha_{n-1}\right| \leq N} a_{\alpha_{1}, \ldots, \alpha_{n-1}, 0} e^{i\left(\alpha_{1} t_{1}+\ldots+\alpha_{n-1} t_{n-1}\right)}\right| \\
\leq \max |F(t)| .
\end{array}
$$

Since the trigonometric polynomial

$$
\sum_{\left|\alpha_{1}\right|+\ldots+\left|\alpha_{n-1}\right| \leq N} a_{\alpha_{1}, \ldots, \alpha_{n-1}, 0} e^{i\left(\alpha_{1} t_{1}+\ldots+\alpha_{n-1} t_{n-1}\right)}
$$

is real and its modulus does not exceed

$$
\max |F(t)|-\left|a_{N e_{n}}\right|-\left|a_{-N e_{n}}\right|,
$$

we apply the result for dimension $n-1$ and complete the proof.

3. An estimate of the Bohr radius. We are now going to apply the inequality (2.2) to the estimation from below of the Bohr radius for the hypercone $\mathcal{D}_{1}^{n}$.

Consider the homogeneous polynomial of degree $k$ in $n$ complex variables

$$
P_{k}(z)=\sum_{|\alpha|=k} c_{\alpha} z^{\alpha}, \quad k=1,2, \ldots
$$


satisfying

$$
\left|P_{k}(z)\right| \leq 1, \quad z \in \overline{\mathcal{D}_{1}^{n}}
$$

For such homogeneous polynomials, define

$$
A_{k}^{n}=\sup \sum_{|\alpha|=k}\left|c_{\alpha}\right|\left|z^{\alpha}\right|
$$

where the sup is taken not only over $z \in \mathcal{D}_{1}^{n}$ but also over all the homogeneous polynomials of degree $k$ which satisfy (3.1).

Theorem 3.1. We have

$$
A_{k}^{n} \leq \sqrt{\left(\begin{array}{c}
n+k-1 \\
k
\end{array}\right)-n} \sqrt{1-n^{-2 k}(3 n-2)}+n^{1-k}, \quad k=1,2, \ldots
$$

Proof. From the assumption (3.1) it follows that

$$
\left|P_{k}\left(\frac{1}{n}, \frac{1}{n} e^{i t_{2}}, \ldots, \frac{1}{n} e^{i t_{n}}\right)\right| \leq 1 \text {. }
$$

Therefore

$$
n^{2 k}\left|P_{k}\left(\frac{1}{n}, \frac{1}{n} e^{i t_{2}}, \ldots, \frac{1}{n} e^{i t_{n}}\right)\right|^{2} \leq n^{2 k} .
$$

The left-hand side of (3.3) is a trigonometric polynomial in $n-1$ variables. This polynomial is obviously real and is equal to

$$
\begin{array}{r}
\sum_{|\alpha|=k}\left|c_{\alpha}\right|^{2}+\sum_{j=2}^{n}\left(\bar{c}_{k e_{1}} c_{k e_{j}} e^{i k t_{j}}+c_{k e_{1}} \bar{c}_{k e_{j}} e^{-i k t_{j}}\right) \\
+ \text { terms with at least two nonzero indices. }
\end{array}
$$

Applying (2.2) we deduce that

$$
\sum_{|\alpha|=k}\left|c_{\alpha}\right|^{2}+2\left|c_{k e_{1}}\right|\left(\left|c_{k e_{2}}\right|+\ldots+\left|c_{k e_{n}}\right|\right) \leq n^{2 k}
$$

Similarly to (3.4) one gets the other $n-1$ inequalities, in which the factor next to 2 is of the type $\left|c_{k e_{j}}\right|$ (i.e. $k$ is at place $j, j=2, \ldots, n-1$ ). Taking the arithmetic mean of those $n$ inequalities we obtain

$$
\begin{aligned}
\sum_{\substack{|\alpha|=k \\
\alpha \in I}}\left|c_{\alpha}\right|^{2}+\frac{n-2}{n}\left(\left|c_{k e_{1}}\right|^{2}+\ldots\right. & \left.+\left|c_{k e_{n}}\right|^{2}\right) \\
& +\frac{2}{n}\left(\left|c_{k e_{1}}\right|+\ldots+\left|c_{k e_{n}}\right|\right)^{2} \leq n^{2 k},
\end{aligned}
$$

where $I=\left\{\alpha \in \mathbb{N}^{n}: \alpha_{j} \neq k, j=1, \ldots, n\right\}$. The inequality (3.5) and a 
classical inequality imply that

$$
\sum_{\substack{|\alpha|=k \\ \alpha \in I}}\left|c_{\alpha}\right|^{2}+\frac{3 n-2}{n^{2}}\left(\left|c_{k e_{1}}\right|+\ldots+\left|c_{k e_{n}}\right|\right)^{2} \leq n^{2 k} .
$$

Note that we pass from (3.5) to (3.6) in order to make the subsequent calculations easier. Proceeding with (3.5) cannot improve the final result.

Furthermore, one observes that the modulus of the polynomial $P_{k}(z)$ does not exceed 1 on the sections of the hypercone by coordinate hyperplanes $\left\{z \in \mathbb{C}^{n}: z_{l}=0, l=1, \ldots, j-1, j+1, \ldots, n\right\}, j=1, \ldots, n$. That is, in the disks $\left\{z_{j} \in \mathbb{C}:\left|z_{j}\right|<1\right\}$ one has $\left|P_{k}(z)\right| \leq 1$. Therefore

$$
\left|c_{k e_{j}}\right| \leq 1, \quad j=1, \ldots, n .
$$

Now, we deduce that

$$
A_{k}^{n} \leq B_{k}^{n}=\sup \sum_{|\alpha|=k}\left|c_{\alpha}\right| x^{\alpha},
$$

where the sup is taken over all $\left(x_{1}, \ldots, x_{n}\right)$ with $x_{j} \geq 0, j=1, \ldots, n$, and $x_{1}+\ldots+x_{n}=1$ and over all $\left|c_{\alpha}\right|$ satisfying (3.6) and (3.7) at the same time.

Since $B_{k}^{n}$ is homogeneous with respect to $\left|c_{\alpha}\right|$, we may consider

$$
\sum_{\substack{|\alpha|=k \\ \alpha \in I}}\left|c_{\alpha}\right|^{2}+\frac{3 n-2}{n^{2}} A^{2}=n^{2 k}
$$

instead of (3.6), where $A=\sum_{j=1}^{n}\left|c_{k e_{j}}\right|$. Ignoring (3.7) for a moment, we arrive at a conditional extremum problem with respect to the variables $\left|c_{\alpha}\right|$, $\alpha \in I$, and $x$. It can be easily solved by using the Lagrange multiplier method. First, the maximum is achieved for $x_{1}=\ldots=x_{n}=1 / n$, and thus $x^{\alpha}=n^{-k}$, and, secondly, all $\left|c_{\alpha}\right|, \alpha \in I$, are equal. Since the number of these $\left|c_{\alpha}\right|$ is $\left(\begin{array}{c}n+k-1 \\ k\end{array}\right)-n$ (see, e.g., [12, Ch. IV, §2]), we obtain

$$
B_{k}^{n}=n^{-k} \sqrt{\left(\begin{array}{c}
n+k-1 \\
k
\end{array}\right)-n} \sqrt{n^{2 k}-\frac{3 n-2}{n^{2}} A^{2}}+n^{-k} A .
$$

It is a routine matter to show that $B_{k}^{n}$ increases in $A$. Recalling (3.7), we take $A=n$ and readily arrive at (3.2). This completes the proof of the theorem.

4. Applications. In the proof of Theorem 9 in [2] it was shown that

$$
A_{k}^{n} \leq k^{k} / k !
$$

The advantage of the estimate (4.1) is that it is independent of the number $n$ of independent variables. At the same time, (3.2) for small $n$ sometimes gives a better bound than (4.1), for example, for $n=2$ and $k=2,3,4,5$, even 
though there are cases where the converse is true: if $n=4$ and $k=2$, then (4.1) gives the bound of 2 while (3.2) gives the bound of 2.6511. Therefore the following corollary might be of importance.

Corollary 4.1.

$$
A_{k}^{n} \leq \min \left(\sqrt{\left(\begin{array}{c}
n+k-1 \\
k
\end{array}\right)-n} \sqrt{1-n^{-2 k}(3 n-2)}+n^{1-k}, \frac{k^{k}}{k !}\right) .
$$

This result allows one in some cases to improve the estimate from below in (1.1), that is,

$$
R\left(\mathcal{D}_{1}^{n}\right)>\frac{1}{3 \sqrt[3]{e}}
$$

The right-hand side is approximately equal to 0.238844 . We remark here that in [8] the following estimate was obtained for $n>1$ :

$$
R\left(U^{n}\right)>\frac{2}{5 \sqrt{n}}
$$

from which the same estimate holds for any complete Reinhardt domain, in particular, for the hypercone:

$$
R\left(\mathcal{D}_{1}^{n}\right)>\frac{2}{5 \sqrt{n}} .
$$

The estimate (4.4) is better than (4.3) for $n=2$, but weaker if $n>2$. With the help of inequality (1.2), in [1] the estimate (3.2) is given for $n=2$ :

$$
R\left(\mathcal{D}_{1}^{2}\right)>0.304236
$$

which is sharper than (4.3) and (4.4).

Similarly, we will now use Theorem 3.1 in order to improve, for some cases when $n>2$, the estimate (4.3) (and therefore (4.4) since (4.3) is better than (4.4) for $n>2$ ).

Corollary 4.2. The following estimates hold:

$$
\begin{array}{rlrl}
R\left(\mathcal{D}_{1}^{3}\right) & >0.271114, & R\left(\mathcal{D}_{1}^{4}\right)>0.258685, & R\left(\mathcal{D}_{1}^{5}\right)>0.251975, \\
R\left(\mathcal{D}_{1}^{6}\right)>0.247485, & R\left(\mathcal{D}_{1}^{7}\right)>0.245426, & R\left(\mathcal{D}_{1}^{8}\right)>0.243525, \\
R\left(\mathcal{D}_{1}^{9}\right)>0.242473, & R\left(\mathcal{D}_{1}^{10}\right)>0.241522 . &
\end{array}
$$

Proof. Let $f(z)=\sum_{|\alpha| \geq 0} c_{\alpha} z^{\alpha}$ be a holomorphic function in the hypercone $\mathcal{D}_{1}^{n}$ with $|f(z)|<1$ for $z \in \mathcal{D}_{1}^{n}$. Then, as shown in [1], for $k \geq 1$ we have the Cauchy-Landau type estimate

$$
\left|\sum_{|\alpha|=k} c_{\alpha} z^{\alpha}\right|<2\left(1-\left|c_{0, \ldots, 0}\right|\right), \quad z \in \mathcal{D}_{1}^{n} .
$$


Furthermore, using Corollary 4.1, one has

$$
\sum_{\substack{|\alpha| \geq 0 \\|+\ldots+| z_{n} \mid \leq x}}\left|c_{\alpha} z^{\alpha}\right|<\left|c_{0, \ldots, 0}\right|+2\left(1-\left|c_{0, \ldots, 0}\right|\right) \sum_{k=1}^{\infty} C_{k}^{n} x^{k},
$$

where $C_{k}^{n}$ denotes the right-hand side in (4.2). Hence it can be readily seen that $R\left(\mathcal{D}_{1}^{n}\right) \geq x_{k}$, where $x_{k}$ is the root of the equation

$$
\sum_{k=1}^{\infty} C_{k}^{n} x^{k}=1 / 2 .
$$

After solving numerically the equation (4.5) for $n=3, \ldots, 10$ by using MAPLE we find the results claimed in the statement of Corollary 4.2.

Concluding, we remark that for sufficiently large $n$ the min in (4.2) is equal to the right-hand side of (4.1) and we again return to the earlier estimate (4.3).

REMARK 4.1. The bounds of the Bohr radius from Corollary 4.2 also hold for every Reinhardt domain of the type

$$
D=\left\{z \in \mathbb{C}^{n}: \Phi\left(\left|z_{1}\right|, \ldots,\left|z_{n}\right|\right)<0\right\},
$$

where $\Phi$ is a convex function, that is, $D$ is a union of hypercones

$$
\left\{z \in \mathbb{C}^{n}: a_{1}\left|z_{1}\right|+\ldots+a_{n}\left|z_{n}\right|<1\right\} .
$$

\section{References}

[1] L. Aizenberg, Generalization of Carathéodory's inequality and the Bohr radius for multidimensional power series, Complex Variables, to appear.

[2] - Multidimensional analogues of Bohr's theorem on power series, Proc. Amer. Math. Soc. 128 (2000), 1147-1155.

[3] -, Bohr Theorem, in: Encyclopedia of Mathematics, Supplement II, M. Hazewinkel (ed.), Kluwer, Dordrecht, 2000, 76-78.

[4] L. Aizenberg, A. Aytuna and P. Djakov, An abstract approach to Bohr's phenomenon, Proc. Amer. Math. Soc. 128 (2000), 2611-2619.

[5] - - - - , Generalization of Bohr's theorem for bases in spaces of holomorphic functions of several complex variables, J. Math. Anal. Appl. 258 (2001), 428-447.

[6] L. Aizenberg and N. Tarkhanov, A Bohr phenomenon for elliptic equations, Proc. London Math. Soc. 82 (2001), 385-401.

[7] H. P. Boas, Majorant series, J. Korean Math. Soc. 37 (2000), 321-337.

[8] H. P. Boas and D. Khavinson, Bohr's power series theorem in several variables, Proc. Amer. Math. Soc. 125 (1997), 2975-2979.

[9] H. Bohr, A theorem concerning power series, Proc. London Math. Soc. 13 (1914), $1-5$.

[10] J. G. van der Corput and C. Visser, Inequalities concerning polynomials and trigonometric polynomials, Nederl. Akad. Wetensch. Proc. 49 (1946), 383-392. 
[11] P. B. Djakov and M. S. Ramanujan, A remark on Bohr's theorem and its generalizations, J. Analysis 8 (2000), 65-77.

[12] E. M. Stein and G. Weiss, Introduction to Fourier Analysis on Euclidean Spaces, Princeton Univ. Press, Princeton, 1971.

Department of Mathematics and Computer Science

Bar-Ilan University

52900 Ramat-Gan, Israel

E-mail: aizenbrg@macs.biu.ac.il

liflyand@macs.biu.ac.il
Department of Mathematics and Statistics University of Cyprus Nicosia 1678, Cyprus E-mail: msvidras@ucy.ac.cy 